EPiC Series in Language and Linguistics
Volume 1, 2016, Pages 170-180
CILC2016. 8th International
Conference on Corpus Linguistics

\title{
The Poetic Word of Fernando de Herrera. An Approach through Corpus and Computational Linguistics
}

\author{
Laura Hernández Lorenzo ${ }^{1 *}$ \\ ${ }^{1}$ University of Seville, Spain. \\ lhernandez1@us.es
}

\begin{abstract}
Great advances in Corpus Linguistics have led to new approaches in Literary Studies. This paper applies these new tools to the analysis of Golden Age Spanish poetry written by Fernando de Herrera, the author of Anotaciones a Garcilaso de la Vega (1580) and one of the greatest poets of his time. Through a keyword method combined with lexical concordances, we will try to overview principal characteristics and differences between subgenres in Herrera's poetry, dealing with the poems he published in life (known as $H$ ) and getting results which help in the academic debate about this poet's works and style.
\end{abstract}

\section{Corpus Linguistics and Literature Studies}

Corpus Linguistics and Computational tools have been applied to a relevant amount of text types and corpora throughout the recent decades, leading to great advances in the exploration of texts (McEnery \& Wilson, 2005). However, the application of these methodologies and tools to literary texts, and most especially to poetry, is much more infrequent. Most of the contributions in this field have been covered by the discipline of Corpus Stylistics, with a great deal of publications that study English Literature through Stylistics and Corpus Studies methodologies (McIntyre \& Busse, 2010). Furthermore, some other relevant research has been produced by Computational Linguistics research groups with an interest in Literature, published in the last volume of Linguistic Issues in Language Technology with the title Computational Linguistics for Literature in October 2015 (Elson, Feldman, Kazantseva, \& Szpakowicz, 2015). Therefore, this kind of literary analysis that follows the new perspectives of 'macroanalysis' (Jockers, 2013) or 'distant reading' (Moretti, 2007) requires more studies dealing with a subject which reflects and enriches the digital turn in Literature Studies.

\footnotetext{
* FPU Spanish researcher and teaching fellowship. This piece of work is part of a PhD project funded by FPU ('Formación de Profesorado Universitario') Spanish research and teaching fellowship from the Spanish Ministry of Education.
}

A.Moreno Ortiz and C.Pérez-Hernández (eds.), CILC2016 (EPiC Series in Language and Linguistics, vol. 1), pp. $170-180$ 
Research in this field may benefit from the appliances of new technologies and their possibilities to study writers' language through a linguistic-stylistic approach and using as corpora the works of one or more authors. In addition to a more effective extraction of data, corpus and computing tools allow researchers to study Literature working with empiric data and within the context of the scientific method (McEnery \& Hardie, 2012).

When approaching Spanish Literature, corpus and computing-based literary studies is a path almost unexplored, since most of Literature digital humanists work in digital editions (López Poza, 2015). Nevertheless, in the case of works suffering from textual transmission problems, a previous analysis of language and style is needed, as it occurs with Fernando de Herrera's poetic works.

\section{The author: Fernando de Herrera}

Fernando de Herrera (1534-1597) ${ }^{\dagger}$ is one of the most important poets of the Spanish Golden Age, as well as the most influential writer from the group of Sevillian humanists in the second half of the sixteenth century known as the Sevillian Poetic School. Among his works, it is fairly to name Obras de Garcilaso de la Vega con Anotaciones de Fernando de Herrera or Works of Garcilaso de la Vega with Comments by Fernando de Herrera (1580) and Algunas Obras or Some Works (1582). In the first one, Herrera -through annotating and commenting on Garcilaso's poems- established his own poetic theory. Then, he published Algunas Obras, a collection of his own poetry carefully selected and prepared, in which he put into practice the theories about poetry and language that he had defended earlier.

On the top of the importance that it had at the time, Herrera's poetry has also been considered especially by Dámaso Alonso and Antonio Vilanova- of great relevance in understanding the evolution of poetic language from the Renaissance writing of Garcilaso de la Vega (1501-1536) to the baroque style of Luis de Góngora (1561-1627). However, the transmission of Herrera's poetry to the present includes another poetry edition and some manuscript poems. The matter consists of the big and significant differences between poems published in Herrera's life and those included in this second edition, titled Versos de Fernando de Herrera or Verses of Fernando de Herrera and published in 1619 after the poet's death by his admirer, the Sevillian painter Francisco Pacheco (15641644). This circumstance has provoked an academic debate between researchers and experts which is known as the 'textual drama' of Herrera's poetry. Besides, Herrera's tendency to correct his poems over and over again in a desire for perfection has added another difficulty to the problem. In order to shed light on the discussion, this study provides a linguistic-stylistic analysis of Herrera's poetic language based on Corpus Linguistics tools. Its final goal is to get empiric data which enriches classic and fundamental studies as those by Macrí (1972) and Kossoff (1966). To achieve this, the present study centres in the particular and distinguished features of Herrera's varied genres of poetry through a keyword analysis.

\section{Compilation of the corpus}

The corpora used in this study are the poems that Herrera published during his life in Algunas Obras, also known as $H$. This printed edition is considered by experts almost as an authoritative autograph, since we have evidence of Herrera's participation on the printing process, even revising print proofs, something that was not very usual in the sixteenth century printing houses. Hitherto, it is

\footnotetext{
${ }^{\dagger}$ Very interesting digital materials about this writer and some introductory information on his life and works, as well as a compilation of relevant studies, can be found in the section devoted to him in the Biblioteca Virtual Miguel de Cervantes. URL: http://www.cervantesvirtual.com/portales/fernando_de_herrera/
} 
the only edition of Herrera's poetry completely reliable in terms of text and authorship. In the present study, the ninety-one poems that it contains have been separated into different sub corpora which reflect the diverse and various minus genres of his poetry.

A total of four sub corpora corresponding with the four minus genres in Herrera's poems included in Algunas Obras have been used. These minus genres are sonnets, songs, elegies and a hunting eclogue. As it can be seen in Table 1, the size of corpora goes from the 7329 words of sonnets, the biggest corpus, to 972 words in the eclogue, the smallest. The number of poems also varies depending on the genre. The vast majority of poems included in Algunas Obras are sonnets, with a total of 78 poems. Songs and elegies appear less frequently in the book, and in the case of the hunting eclogue, there is only one poem, though very extensive.

\begin{tabular}{lccc}
\hline & Corpora & Poems & Words \\
\hline Sonnets & SON & 78 & 7329 \\
Songs & CAN & 5 & 2871 \\
Elegies & ELE & 7 & 7494 \\
Hunting Eclogue & EGL & 1 & 972 \\
\hline
\end{tabular}

Table 1: Corpora used classified by poetic genre

In this study, these corpora will be compared and contrasted with a keywords analysis, aided by concordances of the most relevant words in the corpus.

Algunas Obras' entire corpus has been digitalized using OCR (Optical Character Recognition) software in Blecua's annotated edition (Blecua \& Herrera, 1975). After that, the corpus was carefully revised and corrected from the OCR mistakes, which affected especially to the particular punctuation system of the poet. Herrera's language -and most specifically his particular orthography- has not been adapted or modernized, since Unicode UTF-8 encoding allows us to maintain and reproduce it. We think this is interesting since it reflects Herrera's theory about writing and language. The text has not been annotated, as it was not needed for this analysis. Instead, only the poem's titles were annotated in order to avoid interferences with the results.

For this analysis, the software used was AntConc (Anthony, 2014), as it provides the necessary tools as well as works efficiently with the corpora. Keywords were generated using the statistical function log-likelihood, since the corpora we use is relatively small (Rayson \& Garside, 2000).

Keywords were processed in two different ways. Firstly, the rest of the corpora were used as a reference corpus in order to see the main features of the genre. Secondly, when two corpora have similar keywords or a comparison between them was of interest, keywords of each of them were generated using the other as a reference corpus.

\section{Results}

In the sections below the results of the keywords list are shown, classified and shortly commented on. Interpretations and findings will be treated in point 5 .

\subsection{Sonnets}

As it can be seen in Image 1, the principal sonnets' keywords generated using songs, elegies and the hunting eclogue as a reference corpus are: (1) mi, (2) mal, (3) fuego, (4) ielo, (5) dura, (6) error, (7) porque, (8) abráso, (9) acabar, (10) altivo, (11) bellas, (12) blanco, (13) encender, (14) mejor, (15) noble, (16) vale, (17) temo, (18) perdido, (19) alegria, (20) ausente, (21) nunca, (22) fin, (23) antiguo, (24) armas, (25) cessa, (26) desespero, (27) desmaya, (28) encendimiento, (29) esperè, (30) 
esquivo, (31) exemplo, (32) falto, (33) húyo, (34) ora, (35) osa, (36) otomano, (37) perpetua, (38) sois, (40) temi, (41) vo, (42) aunqu, (43) pierdo, (44) remedio, (45) llanto, (46) grave, (47) lagrimas, (48) llóro, (49) principio, (50) suspiros.

\begin{tabular}{|c|c|c|c|c|c|c|c|}
\hline \multicolumn{3}{|c|}{ Types Before Cut: 1855} & \multirow{2}{*}{$\begin{array}{l}\text { Types After Cut: } 1430 \\
\text { Keyword }\end{array}$} & \multicolumn{3}{|c|}{ Types Before Cut: 1855} & \multirow{2}{*}{$\begin{array}{l}\text { Types After Cut: } 1430 \\
\text { Keyword }\end{array}$} \\
\hline Rank & Freq & Keyness & & Rank & Freq & Keyness & \\
\hline 1 & 171 & 27.293 & $\mathrm{mi}$ & 22 & 18 & 6.129 & fin \\
\hline 2 & 47 & 13.967 & mal & 23 & 3 & 5.609 & antiguo \\
\hline 3 & 32 & 12.440 & fuego & 24 & 3 & 5.609 & armas \\
\hline 4 & 18 & 10.988 & ielo & 25 & 3 & 5.609 & cessa \\
\hline 5 & 10 & 9.878 & dura & 26 & 3 & 5.609 & desespero \\
\hline 6 & 19 & 8.135 & error & 27 & 3 & 5.609 & desmaya \\
\hline 7 & 14 & 7.725 & porque & 28 & 3 & 5.609 & encendimiento \\
\hline 8 & 4 & 7.479 & abráso & 29 & 3 & 5.609 & esperè \\
\hline 9 & 4 & 7.479 & acabar & 30 & 3 & 5.609 & esquivo \\
\hline 10 & 4 & 7.479 & altivo & 31 & 3 & 5.609 & exemplo \\
\hline 11 & 4 & 7.479 & bellas & 32 & 3 & 5.609 & fal to \\
\hline 12 & 4 & 7.479 & blanco & 33 & 3 & 5.609 & húyo \\
\hline 13 & 4 & 7.479 & encender & 34 & 3 & 5.609 & ora \\
\hline 14 & 4 & 7.479 & mejor & 35 & 3 & 5.609 & osa \\
\hline 15 & 4 & 7.479 & noble & 36 & 3 & 5.609 & otomano \\
\hline 16 & 8 & 6.944 & vale & 37 & 3 & 5.609 & perpetua \\
\hline 17 & 6 & 6.474 & temo & 38 & 3 & 5.609 & sois \\
\hline 18 & 14 & 6.423 & perdido & 39 & 3 & 5.609 & temi \\
\hline 19 & 9 & 6.323 & alegria & 40 & 3 & 5.609 & umor \\
\hline 20 & 9 & 6.323 & ausente & 41 & 3 & 5.609 & vo \\
\hline 21 & 17 & 6.164 & nunca & 42 & 7 & 5.548 & aunqu \\
\hline
\end{tabular}

Figure 1: SON keywords. Corpus reference: CAN, ELE and EGL.

This data can be classified in the following way:

1. Abundance and relevance of grammatical first person pronouns -mi, me, $m, m i s, y o-$ and verbal forms -veo, estoi, voi, muero, pierdo, buelvo, sè, temo, llóro, abráso...

2. Words related with love suffering -error, mal, acabar, perdido, ausente, desespero, llanto, lágrimas, grave, suspiros, daño, dolor, llóro.

3. Words related with lady's coldness and beauty -ielo, blancos, bellos.

4. Importance of the adverb nunca and the verb form vale. The first one is related with feelings, a painful denial and forever, whereas vale stands for love complaints and helplessness.

5. Words related with the metaphor of love passion as a fire are especially relevant (fuego, encender, ardiente, encendimiento, abrasar). It is interesting how they increase in keyness when the keyword list is generated with the elegies as a reference corpus: (1) fuego, (13) abráso, (14) encender, (30) ardiente, (40) encendimiento. 


\subsection{Elegies}

As it can be seen in Image 2, the main elegies' keywords generated using songs, sonnets and the hunting eclogue as a reference corpus are: (1) me, (2) no, (3) una, (4) cuan, (5) bienes, (6) flechas, (7) hallar, (8) breve, (9) es, (10) puedo, (11) tan, (12) causa, (13) yo, (14) animo, (15) e, (16) espacio, (17) fui, (18) mesquino, (19) premio, (20) puedes, (21) quiero, (22) umano, (23) voluntad, (24) algun, (25) dezir, (26) igual, (27) siento, (28) agena, (29) alabança, (30) ambicion, (31) cerca, (32) condena, (33) desconcierto, (34) firmeza, (35) gozar, (36) larga, (37) ledo, (38) levánte, (39) mirar, (40) padece, (41) pensar, (42) reposo, (43) suele, (44) valio, (45) venid, (46) bien, (47) ni, (48) m, (49) està, (50) quien.

\begin{tabular}{|c|c|c|c|c|c|c|c|}
\hline \multicolumn{3}{|c|}{ Types Before Cut: 1942} & \multirow{2}{*}{$\begin{array}{l}\text { Types After Cut: } 1504 \\
\text { Keyword }\end{array}$} & \multicolumn{3}{|c|}{ Types Before Cut: 1942} & \multirow{2}{*}{$\begin{array}{l}\text { Types After Cut: } 1504 \\
\text { Keyword }\end{array}$} \\
\hline Rank & Freq & Keyness & & Rank & Freq & Keyness & \\
\hline 1 & 90 & 17.464 & me & 22 & 4 & 7.301 & umano \\
\hline 2 & 135 & 10.692 & no & 23 & 4 & 7.301 & voluntad \\
\hline 3 & 12 & 9.970 & una & 24 & 8 & 6.647 & algun \\
\hline 4 & 13 & 9.284 & cuan & 25 & 8 & 6.647 & dezir \\
\hline 5 & 5 & 9.126 & bienes & 26 & 8 & 6.647 & igual \\
\hline 6 & 5 & 9.126 & flechas & 27 & 10 & 5.607 & siento \\
\hline 7 & 5 & 9.126 & hallar & 28 & 3 & 5.476 & agena \\
\hline 8 & 11 & 8.609 & breve & 29 & 3 & 5.476 & alabança \\
\hline 9 & 46 & 8.463 & es & 30 & 3 & 5.476 & ambicion \\
\hline 10 & 15 & 8.410 & puedo & 31 & 3 & 5.476 & cerca \\
\hline 11 & 28 & 8.006 & $\tan$ & 32 & 3 & 5.476 & condena \\
\hline 12 & 7 & 7.775 & causa & 33 & 3 & 5.476 & desconcier to \\
\hline 13 & 47 & 7.345 & yo & 34 & 3 & 5.476 & firmeza \\
\hline 14 & 4 & 7.301 & animo & 35 & 3 & 5.476 & gozar \\
\hline 15 & 4 & 7.301 & e & 36 & 3 & 5.476 & larga \\
\hline 16 & 4 & 7.301 & espacio & 37 & 3 & 5.476 & ledo \\
\hline 17 & 4 & 7.301 & fui & 38 & 3 & 5.476 & levánte \\
\hline 18 & 4 & 7.301 & mesquino & 39 & 3 & 5.476 & mirar \\
\hline 19 & 4 & 7.301 & premio & 40 & 3 & 5.476 & padece \\
\hline 20 & 4 & 7.301 & puedes & 41 & 3 & 5.476 & pensar \\
\hline 21 & 4 & 7.301 & quiero & 42 & 3 & 5.476 & reposo \\
\hline
\end{tabular}

Figure 2: ELE keywords. Corpus reference: SON, CAN and EGL.

This data can be classified in the following way:

1. Abundance of verbs in present tense -es, puedo, puedes, quiero, siento, padece, suele, està, pienso, alcança.

2. Presence of strong denial $-n o$ and $n i$.

3. Abundance of first person verbs and pronouns -comigo, fui, quiero, me, yo, puedo, quiero, siento, pienso, $m$.

4. Vocabulary related with intimacy, interiority and feelings -animo, umano, voluntad.

5. The appearance of the second person besides other words addressing the hearer/beloved become more relevant when sonnets are used as the only reference corpus for elegies' 
keywords -señora, te, contigo, puedes, tienes, te, as. Therefore, elegies in general, and especially when contrasted with sonnets, can be considered as missive poems.

\subsection{Songs}

As it can be seen in Image 3, top songs' keywords generated using elegies, sonnets and the hunting eclogue as a reference corpus are: (1) su, (2) con, (3) las, (4) valor, (5) $i$, (6) sobre, (7) generosa, (8) iupiter, (9) sobervia, (10) vanderas, (11) gloriöso, (12) marte, (13) ramos, (14) los, (15) cielo, (16) lira, (17) sangre, (18) vigor, (19) rayo, (20) nombre, (21) acabo, (22) aves, (23) cantava, (24) cervizes, (25) cubrio, (26) doblado, (27) elicona, (28) eroica, (29) esclarecida, (30) espantoso, (31) estima, (32) famosos, (33) fatal, (34) fuertes, (35) impios, (36) lança, (37) linage, (38) lusitania, (39) minerva, (40) ombres, (41) ondosa, (42) osados, (43) peloro, (44) reinos, (45) religion, (46) resonante, (47) sonora, (48) sublima, (49) sustenta, (50) tifeo.

\begin{tabular}{|c|c|c|c|c|c|c|c|}
\hline \multicolumn{3}{|c|}{ Types Before Cut: 1160} & \multirow{2}{*}{$\begin{array}{l}\text { Types After Cut: } \\
\text { Keyword }\end{array}$} & \multicolumn{3}{|c|}{1001 Types Before Cut: 1160} & \multirow{2}{*}{$\begin{array}{l}\text { Types After Cut: } 1001 \\
\text { Keyword }\end{array}$} \\
\hline Rank & Freq & Keyness & & Rank & Freq & Keyness & \\
\hline 1 & 47 & 25.135 & su & 22 & 2 & 7.488 & aves \\
\hline 2 & 54 & 21.469 & con & 23 & 2 & 7.488 & cantava \\
\hline 3 & 32 & 14.688 & las & 24 & 2 & 7.488 & cervizes \\
\hline 4 & 15 & 14.274 & valor & 25 & 2 & 7.488 & cubrio \\
\hline 5 & 191 & 13.879 & i & 26 & 2 & 7.488 & doblado \\
\hline 6 & 5 & 13.648 & sobre & 27 & 2 & 7.488 & elicona \\
\hline 7 & 3 & 11.232 & generosa & 28 & 2 & 7.488 & eroica \\
\hline 8 & 3 & 11.232 & iupiter & 29 & 2 & 7.488 & esclarecida \\
\hline 9 & 3 & 11.232 & sobervia & 30 & 2 & 7.488 & espantoso \\
\hline 10 & 3 & 11.232 & vanderas & 31 & 2 & 7.488 & estima \\
\hline 11 & 5 & 11.013 & gloriöso & 32 & 2 & 7.488 & famosos \\
\hline 12 & 6 & 10.340 & marte & 33 & 2 & 7.488 & fatal \\
\hline 13 & 4 & 10.306 & ramos & 34 & 2 & 7.488 & fuertes \\
\hline 14 & 29 & 9.898 & los & 35 & 2 & 7.488 & impios \\
\hline 15 & 18 & 8.907 & cielo & 36 & 2 & 7.488 & lança \\
\hline 16 & 4 & 8.006 & lira & 37 & 2 & 7.488 & linage \\
\hline 17 & 4 & 8.006 & sangre & 38 & 2 & 7.488 & lusitania \\
\hline 18 & 4 & 8.006 & vigor & 39 & 2 & 7.488 & minerva \\
\hline 19 & 6 & 7.833 & rayo & 40 & 2 & 7.488 & ombres \\
\hline 20 & 8 & 7.763 & nombre & 41 & 2 & 7.488 & ondosa \\
\hline 21 & 2 & 7.488 & acabò & 42 & 2 & 7.488 & osados \\
\hline
\end{tabular}

Figure 3: CAN keywords. Reference corpus: SON, ELE and EGL.

This data can be classified in the following way:

1. Third person possessive pronoun $s u$ is the highest word in the keyword list, regardless the reference corpus used to generate it.

2. Determinative articles have a special relevance-los, las, la.

3. Abundance of third person singular verbs -acabò, cantava, cubrio, viste, alçarà.

4. Prepositions con and sobre are on the top of the keywords list. In this case, it is interesting to see the concordances or KWIC (Keyword in Context) of each of them in Image 4 and 5. 


\begin{tabular}{|c|c|c|c|}
\hline \multirow{2}{*}{\multicolumn{3}{|c|}{$\begin{array}{l}\text { Concordance Hits } 5 \\
\text { Hit KWIC }\end{array}$}} & \multirow[b]{2}{*}{ File } \\
\hline & & & \\
\hline 1 & & - i con terrible espanto el Señor visitò sobre sus males, 35 para umillar los fuertes arro & CANTXT \\
\hline 2 & & ndinados las ardientes espadas desnudar on sobre la claridad $i$ hermosura de tu gloria & CAN.TXT \\
\hline 3 & & a alteza; las aguas lo criaron poder oso, 70 sobre empinados arboles subido, i se multipli & CANTXT \\
\hline 4 & & estima lo qu'a virtud conviene; i sobre cuanto precia 85 el vulgo incierto, sui & CAN.TXT \\
\hline 5 & & o, i con doradas alas la Vitoria sobre el orbe del fuego, resonando su gloria & CAN.TXT \\
\hline
\end{tabular}

Figure 4: Sobre KWIC

\begin{tabular}{|c|c|c|c|}
\hline \multirow{2}{*}{\multicolumn{3}{|c|}{$\begin{array}{l}\text { Concordance Hits } 54 \\
\text { Hit KWIC }\end{array}$}} & \multirow{3}{*}{$\begin{array}{l}\text { File } \\
\text { CAN.TXT }\end{array}$} \\
\hline & & & \\
\hline 1 & & de gloria. i la llorosa istoria assómbre con orror funesto i triste, dend'el Africo & \\
\hline 2 & & a aquella cumbre d'eterna luz; mas con sobervia cierta 20 se ofrecier on la incierta & CAN.TXT \\
\hline 3 & & , i sin bolver a Dios sus ojos, con ier to cuello i coraçon ufano solo atendier & CAN.TXT \\
\hline 4 & & nuevo Sol, presago de mal tanto. i con terrible espanto el Señor visitò sobre sus & CAN.TXT \\
\hline 5 & & ; i levantò los barbaras no iguales, que con osados pechos i constantes no busquen oro: & CAN.TXT \\
\hline 6 & & pechos $\mathrm{i}$ constantes no busquen oro; mas con crudo hierro venguen la ofensa i cometido & CAN.TXT \\
\hline 7 & & earon 45 mesquina Lusitania sin ventura. i con frente segura rompieron sin temor con fie & CAN.TXT \\
\hline 8 & & $\mathrm{i}$ con frente segura rompieron sin temor con fiero estrago tus armadas escuadras $\mathrm{i} b \mathrm{br}$ & CAN.TXT \\
\hline 9 & & arena se tornò sangriento lago, 50 la llanura con muertos aspereza. cayò en unos vigor, cay & CAN.TXT \\
\hline 10 & & fuertes i belíger os var ones, 55 que conturbar on con furor la tierra? que sacudier on reinos pod & CAN.TXT \\
\hline 11 & & del al to Libano, vestido de ramos, hojas, con ecelsa alteza: las aguas lo criaron poder & CAN.TXT \\
\hline 12 & & i se multiplicaron en grandeza sus ramos con belleza; i, estendiendo su sombra, s'anida & CAN.TXT \\
\hline 13 & & arbol alguno a su figura. Pero elevóse con su verde cima, 80 i sublimò la presuncion & CAN.TXT \\
\hline 14 & & 00 alguna vez el Español corage, despedaçada con aguda lança compensaràs muriendo el hec & CAN.TXT \\
\hline 15 & & rible, i el Lusitano orgullo quebrantado con estrago increible; que no menor corona & CAN.TXT \\
\hline 16 & & cervizes umilladas presas en cativerio con vencedora mano; 45 ni que de las vander as & CAN.TXT \\
\hline 17 & & s vanderas ensalçadas el Cita i Africano con medroso semblante, i el Indo i Persa & CAN.TXT \\
\hline 18 & & amor enel fuego, viendo su bella luz, con fuerça nueva, 70 sin admitir sossiego, & CAN.TXT \\
\hline 19 & & uestra abier to 100 el bien, a otros secreto, con guia tal, qu'enel peligro oscuro de & CAN.TXT \\
\hline 20 & & Cuando con resonante rayo, i furor del braço poderos & CAN.TXT \\
\hline 21 & & uerra por la sangrienta espada 10 de Marte, con mil muertes no domada; & CAN.TXT \\
\hline 22 & & ; En la celeste cumbre es fama, que con dulce voz presente Febo, autor de la & CAN.TXT \\
\hline
\end{tabular}

Figure 5: Con KWIC.

As can be deducted from concordances, these words appear in narrative and descriptive contexts where they emphasize concrete qualities in the narration.

5. Cielo and lira may seem unrelated to the rest of the keywords at a first glance, but if we generate the concordances, the results are enlightening (see Image 6 and 7). Cielo appears in heroic contexts, although it is frequent in a love song. The element that remains the same in these different contexts is the meaning of 'Providence, God, gods, Divinity'. As a consequence, cielo has the connotation or nuance of what is high and sublime. On the other hand, lira especially appears in love songs, and only one time in a heroic context. Its meaning in all of these texts has to do with poetry and poetic inspiration.

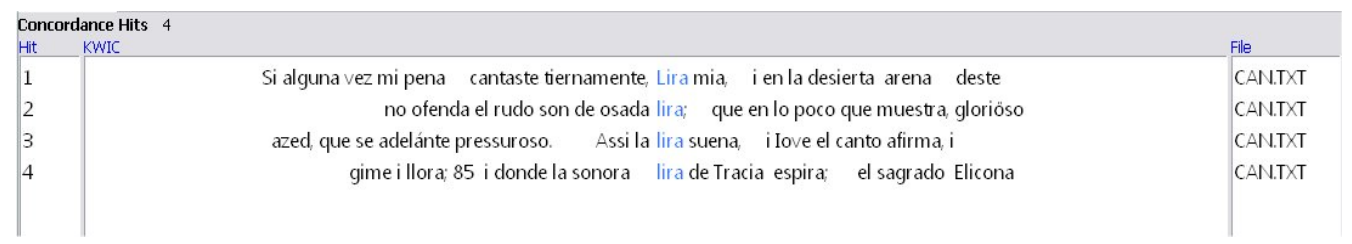

Figure 6: Lira KWIC 


\begin{tabular}{|c|c|c|c|}
\hline \multicolumn{2}{|c|}{ Concordance Hits } & & File \\
\hline 1 & & i de plazer el reino ageno. el cielo no alumbrò, quedò confuso el nuevo Sol, & CANTXT \\
\hline 2 & & 'anidar on las aves, que sustenta el grande cielo; 75 i en sus hojas las fier as engendrar on, & CANTXT \\
\hline 3 & & suelo la que consigo os alçarà enel cielo. No os desvanesca el pecho las & CANTXT \\
\hline 4 & & las divinas Musas vais seguro; do el cielo os muestra abier to $100 \mathrm{el}$ bien, a otros & CANTXT \\
\hline 5 & & suspende atento al immortal senado; i el cielo, que movia su curso arrebatado, $20 \mathrm{~s}$ & CAN.TXT \\
\hline 6 & & a su intento. Cantava la vitoria del cielo, i el orror i l'aspereza que & CANTXT \\
\hline 7 & & ti, dezia, escudo, a tivalor del cielo poderoso, poner temor no pudo el & CANTXT \\
\hline 8 & & mostrarà la luz desta hazaña. Que el cielo le concede de Cesar sacro el ramo & CANTXT \\
\hline 9 & & , $125 \mathrm{i}$ a do iela i abrasa el cielo ardiente. & CAN.TXT \\
\hline 10 & & osadia i arte. Si este valiera al cielo contra el profano exercito arrogante, & CANTXT \\
\hline 11 & & , i s'estremece sacudido, i resuena el cielo, i resplandece, 145 i Mavorte medroso s'oscu & CANTXT \\
\hline 12 & & tendida su grandeza; i vos, lumbres del cielo, mirad felices nuestro Esperio suelo. & CANTXT \\
\hline 13 & & ndo esta serena Estrella alça al rosado cielo, dando alegria al suelo, los ojos, d & CANTXT \\
\hline 14 & & i grandeza, enel dichoso dia 45 qu'el cielo largo la bolvio mas grato. vivo i & CANTXT \\
\hline 15 & & curso de las ondas; i esclareciendo el cielo, 65 esparzio olor suäve en torno el suelo. & CAN.TXT \\
\hline 16 & & su cumbre sostiene la belleza, qu'el cielo en tierra tiene. Yo entretexer quis & CANTXT \\
\hline 17 & & tiernas perlas la ribera ondosa i al cielo alçò la barba revestida de verde musgo & CANTXT \\
\hline 18 & & suelo, un principe guerrero alçar s'al cielo. & CANTXT \\
\hline
\end{tabular}

Figure 7: Cielo KWIC

6. In general, there is a high abundance and predominance of heroic vocabulary -valor, iupiter, sobervia, vanderas, gloriöso, marte, ramos, sangre, vigor, rayo, nombre, eroica, famosos, fuertes, lança, linage, minerva, osados, reinos.

7. If we generate songs' keywords using sonnets and elegies as a reference corpus (or just one of them), the results are very similar.

\subsection{Hunting eclogue}

As it can be seen in Image 8, top hunting eclogue' keywords generated using elegies, sonnets and songs as a reference corpus are: (1) javali, (2) ciervo, (3) ninfa, (4) ven, (5) $t$, (6) te, (7) caçadora, (8) clearista, (9) endimiòn, (10) muera, (11) tu, (12) comigo, (13) bosque, (14) baxa, (15) garça, (16) halcon, (17) lloro, (18) murmurio, (19) ruido, (20) valle, (21) fuente, (22) hermosa, (23) prado, (24) contigo, (25) amante, (26) fiera, (27) agua, (28) arco, (29) braços, (30) caçador, (31) ligera, (32) monte, (33) à, (34) feroz, (35) selva, (36) llama, (37) abites, (38) aborrece, (39) adora, (40) adornára, (41) agradarà, (42) agradasse, (43) alamos, (44) aljava, (45) amarte, (46) apartarà, (47) aprendera, (48) aras, (49) atravessado, (50) blancas.

This data can be classified in the following way:

1. Bucolic and pastoral vocabulary -ninfa, Clearista, Endimiòn, bosque, garça, murmurio, valle, fuente, prado, fiera, agua, monte, selva.

2. Hunting vocabulary -javali, ciervo, caçadora, halcon, arco, caçador, feroz.

3. Abundance and relevance of second person singular pronouns, determiners and verb forms $v e n, t$, te, tu, baxa, contigo, abites. The presence of imperative forms is quite interesting -ven and baxa.

4. Words referred to the nymph -ninfa, ven, te, caçadora, Clearista, hermosa, contigo, agradasse- in contrast with word referred to the lover/speaker-Endimion, muera, comigo, lloro, amante, caçador, adornára, agradarà . 
5. Love vocabulary -ninfa, caçadora, comigo, contigo, hermosura, amante, braços, llama, adora.

6. Verbs are of special interest. In contrast with other genres in which present tenses verb forms were clearly predominant, in eclogue's keywords other verb tenses as imperative, future and subjunctive increase in importance.

\begin{tabular}{|c|c|c|c|c|c|c|c|}
\hline \multicolumn{3}{|c|}{ Types Before Cut: 445} & \multirow{2}{*}{$\begin{array}{l}\text { Types After Cut: } 396 \\
\text { Keyword }\end{array}$} & \multicolumn{3}{|c|}{ Types Before Cut: 445} & \multirow{2}{*}{$\begin{array}{l}\text { Types After Cut: } 396 \\
\text { Keyword }\end{array}$} \\
\hline Rank & Freq & Keyness & & Rank & Freq & Keyness & \\
\hline 1 & 6 & 35.461 & javali & 22 & 4 & 10.822 & hermosa \\
\hline 2 & 4 & 23.641 & ciervo & 23 & 4 & 10.822 & prado \\
\hline 3 & 4 & 23.641 & ninfa & 24 & 3 & 9.734 & contigo \\
\hline 4 & 4 & 23.641 & ven & 25 & 3 & 8.598 & amante \\
\hline 5 & 6 & 19.467 & $t$ & 26 & 3 & 8.598 & fiera \\
\hline 6 & 9 & 19.407 & te & 27 & 2 & 8.108 & agua \\
\hline 7 & 4 & 18.744 & caçadora & 28 & 2 & 8.108 & $\operatorname{arco}$ \\
\hline 8 & 3 & 17.731 & clearista & 29 & 2 & 8.108 & braços \\
\hline 9 & 3 & 17.731 & endimiòn & 30 & 2 & 8.108 & caçador \\
\hline 10 & 3 & 17.731 & muera & 31 & 2 & 8.108 & ligera \\
\hline 11 & 21 & 17.631 & tu & 32 & 3 & 6.915 & monte \\
\hline 12 & 5 & 17.614 & comigo & 33 & 3 & 6.915 & à \\
\hline 13 & 4 & 12.978 & bosque & 34 & 2 & 6.489 & feroz \\
\hline 14 & 2 & 11.820 & baxa & 35 & 2 & 6.489 & selva \\
\hline 15 & 2 & 11.820 & garça & 36 & 5 & 6.340 & Ilama \\
\hline 16 & 2 & 11.820 & halcon & 37 & 1 & 5.910 & abites \\
\hline 17 & 2 & 11.820 & Illoro & 38 & 1 & 5.910 & aborrece \\
\hline 18 & 2 & 11.820 & murmurio & 39 & 1 & 5.910 & adora \\
\hline 19 & 2 & 11.820 & ruido & 40 & 1 & 5.910 & adornára \\
\hline 20 & 2 & 11.820 & valle & 41 & 1 & 5.910 & agradarà \\
\hline 21 & 3 & 11.214 & fuente & 42 & 1 & 5.910 & agradasse \\
\hline
\end{tabular}

Figure 8: EGL keywords. Reference corpus: SON, ELE and CAN.

\section{Findings and interpretation of results}

After showing, briefly classifying and commenting on the keyword results, in this section findings are revealed through interpretation of the results.

First of all, it must be underlined how the keyword results seem to agree with the traditional characterization of poetic genres according to critics and specialists in Herrera's poetry:

El tono celebrativo se emplea fundamentalmente en las canciones, en especial en las de tema patriótico (de paradigmas métricos muy variados, desde la lira hasta la estancia amplia), mientras el tono «élego» más íntimo lo será en las elegías (siempre en tercetos), y también en los sonetos. Estos últimos son de hecho, como en la mayoría de las colecciones poéticas del Siglo de Oro, la base del poemario y soportan la parte principal de los argumentos de amor, aunque también se hagan cargo de otros temas. Finalmente las églogas son el género que más mira a la antigüedad grecolatina, principalmente a Virgilio, aunque esa perspectiva clásica no falta en ninguno de los géneros poéticos cultivados por Herrera, que, junto a Petrarca e imitadores, tiene muy presentes en sus poesías amorosas a los elegíacos latinos (López Bueno \& Montero Delgado). 
Indeed, sonnets and elegies, as can be deducted from keywords, present a predominance of the grammatical first person which highlights the subjectivity and intimate quality of these genres. One of the most relevant features is the abundance of negations -nunca, no and $n i$ - in order to express the irrevocable feelings and terrible pain the speaker suffers. Nevertheless, in the case of Herrera's poetry, there seem to be some differences between these genres. Words related with love suffering are more characteristic of sonnets, but most especially, vocabulary of love passion as a fire stands as a particular feature of the genre. On the other hand, elegies have a presence of grammatical second person which reflects a timid approach to the hearer instead of focusing entirely on the speaker's pain and suffering.

In contrast with the dominant subjectivity and interiority of sonnets and elegies, songs are characterized by a heroic nature, clearly reflected in the keywords, as we have already seen. The narrative character of these poems has a great relevance as poems are less centred on the speaker. Instead, the poem opens itself to the exterior world. This can be observed in the abundance of third person singular as well as articles. This is characteristic of songs in comparison to sonnets and elegies. Furthermore, high or sublime subjects are the principal element of Herrera's songs, as can be seen in keywords and even in the cases of cielo and lira. Therefore, this sense of highness or sublimit acts as the common element between patriotic and love songs.

Moving to the hunting eclogue, this genre differs from the others by the abundance of pastoral and bucolic terms as well as the large amount of references to the harmonic and pleasant nature of 'locus amoenus'. In addition, terms related with hunting reflect the title and subject of the eclogue, where Herrera follows the "Prose VIII" in La Arcadia by Sannazaro and Garcilaso's Eclogue II (lines 176310 ). Words reflect the topic of love, but most importantly, the grammatical second person singular in addition to words referring to the nymph and imperative verb forms have a relevance that is not in the other genres so far. As a summary of the results and findings of this genre revealed above, Herrera's hunting eclogue is the poem less centred on the speaker's love suffering. Moreover, it is possible to observe an attempt of dialogue with the poet's beloved, addressing her through the poem. What's more, the shepherd Endimiòn's aim is to persuade the nymph Clearista so she accepts and corresponds to his love. The poem turns out to be a persuasive discourse that follows the classical conventions of the Latin and Renaissance eclogue. In the same sense, we must underline the presence of subjunctive and future verb tenses which points out the happy and hypothetic future the speaker anticipates and uses to persuade the evasive nymph.

Apart from all of these features in poetic genres, keywords reflect the tension of contraries so characteristic of Herrera's poetry, which has been highlighted by the experts: 'la permanente agonía entre contrarios, entre la razón y el deseo, entre el autoengaño de la esperanza y la certeza de la desilusión' (López Bueno \& Montero Delgado). This tension appears in different ways, the most representative being the opposition between the speaker and his beloved/hearer and the contrast in the expression of feelings and mood -bien, gozar, premio, alabança, esperança against mal, padece, condena, desconcierto.

\section{Conclusions}

In this paper, Fernando de Herrera's poetry has been analysed through Corpus and Computational Linguistics tools. The results have been highly satisfying as different features in poetic genres were reflected in keywords, leading to a characterisation of genre style and Herrera's poetic language which enriches and complements traditional scholarship and studies in this topic.

In the application of digital methods and tools for the study of literature, it has been proved that Corpus tools can lead to advances in this field, even when processing the connotative and metaphoric language of poetry. 
Future work to be undertaken may broaden authors' corpora, including more Renaissance writers and their works, in order to compare Herrera's poetry with poems written by contemporary poets. Results may improve our understanding of Herrera's poetry and style, complement traditional scholarship and contribute to our knowledge of his output.

\section{References}

Anthony, L. (2013). "A Critical Look at Software Tools in Corpus Linguistics". Linguistic Research, 30(2), 141-161.

Anthony, L. (2014). AntConc (Version 3.4.4w) [Computer Software]. Tokyo, Japan.

Blecua, J. M., \& Herrera, F. d. (1975). Obra poética I. Madrid: Real Academia Española.

Chiappini, G. (1985). Fernando de Herrera y la escuela sevillana. Madrid: Taurus.

Cuevas, C., \& Herrera, F. d. (1985). Poesía castellana original completa. Madrid: Cátedra.

Elson, D. K., Feldman, A., Kazantseva, A., \& Szpakowicz, S. (2015). "Literature Lifts Up Computational Linguistics". Linguistic Issues in Language Technology (LiLT), 12(1), 1-4.

Jockers, M. L. (2013). Macroanalysis: digital methods and literary history. Urbana: University of Illinois Press.

Kossoff, A. D. (1966). Vocabulario de la obra poética de Herrera. Madrid: Real Academia Española.

López Bueno, B., \& Montero Delgado, J. (s.f.). "El autor: Vida y obra". Recuperado el 25 de Febrero de 2016, de Portal de autor Fernando de Herrera de la Biblioteca Virtual Miguel de Cervantes: http://www.cervantesvirtual.com/portales/fernando_de_herrera/autor_vida_obra/

López Poza, S. (2015). "Humanidades Digitales y literaturas hispánicas: presente y futuro". Ínsula(822), 3-5.

Macrí, O. (1972). Fernando de Herrera. Madrid: Gredos.

McEnery, T., \& Hardie, A. (2012). Corpus Linguistics: Method, Theory and Practise. Cambridge: Cambridge University Press.

McEnery, T., \& Wilson, A. (2005). Corpus Linguistics. Edinburgh: Edinburgh University Press.

McIntyre, D., \& Busse, B. (2010). Language and Style: in honour of Mick Short. Houndsmills: Palgrave Macmillan.

Moretti, F. (2007). La literatura vista desde lejos. Barcelona: Marbot Ediciones.

Parodi, G. (2008). "Lingüística de corpus: una introducción al ámbito". RLA. Revista de lingüística teórica y aplicada, 46(1), 93-119.

Pedraza, F. (1980). "Fernando de Herrera". En F. Pedraza, \& M. Rodríguez Cáceres, Manual de literatura española. 2. Renacimiento (págs. 399-422). Tafalla: Cénlit.

Rayson, P., \& Garside, R. (2000). "Comparing Corpora using Frequency Profiling". Proceedings of the Workshop Comparing Corpora, held in conjuction with the 38th annual meeting of the Association for Computational Linguistics (ACL 2000). 